\title{
Etude de la valeur alimentaire des pailles de céréales traitées ou non à la soude IV. - Influence du nombre de distributions
de l'aliment concentré
et de l'addition de bicarbonate de sodium
}

\author{
J.P. DULPHY et A. BIENAIME \\ avec la collaboration technique de Madeleine Dudilieu, Marie JAILler, \\ Jacqueline JAMOT, H. BousqueT et L. L'Hotelier \\ I.N.R.A., Laboratoire des Aliments, \\ Centre de Recherches zootechniques et vétérinaires de Theix, \\ F 63110 Beaumont
}

\begin{abstract}
Résumé
La valeur alimentaire des pailles traitées ou non à la soude décroît lorsque la proportion de concentré, en particulier de céréales, dans la ration s'accroît. Nous avons cherché à limiter cet inconvénient de la distribution de céréales en essayant séparément, puis en les combinant, trois techniques :

- la limitation des quantités de paille offertes;

- le fractionnement de l'apport de l'aliment concentré (4 contre 2 repas) ;

- l'addition de bicarbonate de sodium ( $20 \mathrm{~g}$ par animal et par jour).

Pour cela nous avons utilisé une paille avant ou après traitement à la soude, dans. des rations comportant 45 p. 100 de concentré et distribuées à des moutons.

Le fractionnement de l'apport de l'aliment concentré a permis une augmentation faible $(+4,7$ p. 100) mais significative des quantités de paille ingérées.

La limitation des quantités d'aliment distribuées a permis une augmentation très nette de la digestibilité de la paille traitée.

Le fractionnement de l'apport d'aliment concentré a augmenté la digestibilité des rations, mais de façon non significative $(+1,5$ point seulement).

Enfin l'apport de bicarbonate de sodium n'a pas eu d'influence significative sur la digestibilité des rations. régimes.

Nous avons mesuré en outre les caractéristiques du jus de rumen pour les différents

En définitive notre objectif de limiter partiellement la mauvaise utilisation des pailles en présence d'une quantité d'amidon élevée n'a été atteinte que par la limitation des quantités de paille distribuées. Les résultats obtenus sont discutés.
\end{abstract}




\section{Introduction}

Les rations à base de paille, traitée ou non, contiennent souvent une proportion élevée de complément énergétique. Nous avons montré (Dulphy et al., 1982 b) qu'il était souhaitable que ce dernier contienne des aliments riches en parois très digestibles (pulpe de betteraves, ensilage d'herbe de digestibilité élevée) ou peu d'amidon afin d'éviter une diminution importante de la digestibilité de la paille. Cela n'est cependant pas toujours possible et le complément énergétique est souvent constitué de céréales. Pour essayer d'éviter, avec ce type de complément, une mauvaise utilisation digestive de la paille, nous avons essayé séparément, puis en les combinant, trois techniques :

- la limitation des quantités de paille offertes, puisque celle-ci s'était avérée très efficace dans nos essais antérieurs (DuLPHy et al., 1982 a et 1983);

- le fractionnement de l'apport de l'alimentation concentrée, quoique celui-ci n'ait guère donné de résultats sur la digestion de la cellulose dans un essai de OsBourn et al. (1970). Pour ce faire, nous avons distribué le comp!ément en 2 ou en 4 repas par jour ;

- laddition de bicarbonate de sodium qui s'était, en limitant la chute du pH du contenu du rumen, avérée efficace sur la vitesse de digestion de la cellulose (OsBOURN et al., 1970).

Dans ces essais la digestibilité des pailles et de leurs constituants pariétaux a été mesurée sur des moutons et la composition du jus de rumen a, en outre, été déterminée afin de mieux comprendre l'influence des différents facteurs étudiés.

\section{Matériel et méthodes}

Quatre lots de 3 moutons ont reçu à volonté (10 p. 100 de refus), selon le schéma de la figure 1 , un régime constitué, sur la base de la matière sèche, de 55 p. 100 de paille, traitée ou non manuellement par voie humide avec 5 p. 100 de soude, et de 45 p. 100 d'un aliment concentré composé de 66 p. 100 de maïs-grain et de 33 p. 100 de tourteau de soja. Les rations avaient donc une teneur en MAT comprise entre 13,0 et 13,5 p. 100 . L'aliment concentré a été distribué en 2 ou 4 repas par jour, avec ou sans supplément de $20 \mathrm{~g} /$ jour de bicarbonate de sodium. La paille était distribuée à 8 h 30 et 16 h 30 et le concentré à 8 h et 16 h ou à 8 h, 10 h 30,13 h 30 et $16 \mathrm{~h}$.

Douze rations au total ont donc été constituées et ont aussi chacune été distribuées à volonté à 2 moutons supplémentaires fistulisés du rumen sur lesquels nous avons prélevé du jus de rumen $0,1,2,3,4$ et 6 heures après le premier repas de concentré du matin.

Enfin la paille traitée a également été distribuée à 4 lots de 3 moutons en quantité limitée égale à celle ingérée sous forme de paille non traitée, soit 4 traitements supplémentaires.

Les animaux servant aux mesures de digestibilité et d'ingestibilité étaient des béliers castrés, de race $T e x e l$, âgés de 3 ans, pesant $70 \mathrm{~kg}$ en moyenne et maintenus 
en cage à métabolisme. La période durant laquelle ils ont tous reçu de la paille non traitée et l'aliment concentré a servi à la fois à les habituer à la paille et, à la fin de cette période, à les apparier par groupe de 3 .

\begin{tabular}{|c|c|c|c|c|c|c|c|}
\hline \multirow{3}{*}{$\begin{array}{l}\text { Période } \\
\text { Period }\end{array}$} & & \multicolumn{6}{|c|}{$\begin{array}{l}\text { Nombre d'animaux } \\
\text { Number of animals }\end{array}$} \\
\hline & & \multicolumn{4}{|c|}{$\begin{array}{l}\text { Moutons normaux } \\
\text { Normal sheep }\end{array}$} & \multicolumn{2}{|c|}{$\begin{array}{l}\text { Moutons fistulés } \\
\text { Fistulated sheep }\end{array}$} \\
\hline & & 3 & 3 & 3 & 3 & 2 & 2 \\
\hline \multirow{5}{*}{ 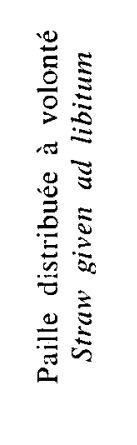 } & $P_{o} \ldots \ldots \ldots$ & PN & PN & PN & $\mathrm{PN}$ & PN & PN \\
\hline & $P_{1}$. & $\begin{array}{l}\text { PN } \\
B^{(2)}\end{array}$ & $\begin{array}{l}\text { PN } \\
(2)\end{array}$ & $\begin{array}{c}\text { PT } \\
\text { B (2) }\end{array}$ & $\begin{array}{l}\text { PT } \\
(2)\end{array}$ & $\begin{array}{l}\text { PN } \\
\text { B (2) }\end{array}$ & $\begin{array}{l}\mathrm{PN} \\
(2)\end{array}$ \\
\hline & $P_{2}, \ldots \ldots \ldots$ & $\begin{array}{r}\text { PN } \\
(4)\end{array}$ & $\begin{array}{l}\text { PN } \\
B(4)\end{array}$ & $\begin{array}{l}\text { PT } \\
(4)\end{array}$ & $\begin{array}{c}\text { PT } \\
\text { B (4) }\end{array}$ & $\begin{array}{l}\text { PN } \\
(4)\end{array}$ & $\begin{array}{l}\text { PN } \\
\text { B (4) }\end{array}$ \\
\hline & $P_{2} \ldots \ldots \ldots$ & $\begin{array}{c}\text { PT } \\
\text { B (2) }\end{array}$ & $\begin{array}{l}\text { PT } \\
(2)\end{array}$ & $\begin{array}{l}\text { PN } \\
\mathrm{B}(2)\end{array}$ & $\begin{array}{l}\mathrm{PN} \\
(2)\end{array}$ & $\begin{array}{l}\text { P' I' } \\
\text { B (2) }\end{array}$ & $\begin{array}{l}\text { PT } \\
(2)\end{array}$ \\
\hline & $P_{4} \ldots \ldots \ldots$ & $\begin{array}{l}\text { PT } \\
(4)\end{array}$ & $\begin{array}{c}\text { PT } \\
\text { B (4) }\end{array}$ & $\begin{array}{l}\text { PN } \\
(4)\end{array}$ & $\begin{array}{l}\text { PN } \\
\text { B (4) }\end{array}$ & $\begin{array}{l}\text { PT } \\
(4)\end{array}$ & $\begin{array}{c}\text { PT } \\
\text { B (4) }\end{array}$ \\
\hline \multirow{2}{*}{ 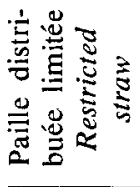 } & $P_{s} \ldots \ldots \ldots$ & $\begin{array}{c}\mathrm{P}^{\mathrm{T}} \\
\mathrm{B}^{(2)}\end{array}$ & $\begin{array}{l}\text { PT } \\
(4)\end{array}$ & $\begin{array}{c}\text { PT } \\
\mathbf{B}(4)\end{array}$ & $\begin{array}{l}\mathrm{PT} \\
(2)\end{array}$ & $\begin{array}{c}\text { PT } \\
\mathrm{B}^{(2)} \\
\end{array}$ & $\begin{array}{l}\text { PT } \\
(4)\end{array}$ \\
\hline & $\mathbf{P}_{6}$ & - & - & - & - & $\begin{array}{l}\text { PT } \\
\text { B (4) }\end{array}$ & $\begin{array}{l}\text { PT } \\
(2)\end{array}$ \\
\hline \multicolumn{8}{|c|}{$\begin{array}{l}\text { PN : Paille normale non traitée - Untreated straw. } \\
\text { PT : Paille traitée - Treated straw. } \\
\text { (B) : Bicarbonate de sodium }(20 \mathrm{~g} / \text { jour }) \text { - Sodium bicarbonate }(20 \mathrm{~g} / \text { day }) . \\
\text { (2) (4) : Nombre de distributions de concentré par jour - Number of concentrate distributions per } \\
\text { day. }\end{array}$} \\
\hline
\end{tabular}

FIG. 1

Schéma expérimental de l'essai.

Scheme of the trial.

Les méthodes danalyse des aliments, des fèces et du jus de rumen ont déjà été rapportées (Dulphy et al., 1982 a et 1983). La composition des pailles utilisées figure dans le tableau 1. 


\section{TABleau 1}

Composition des aliments utilisés.

Chemical composition of the feeds.

\begin{tabular}{|c|c|c|c|c|c|c|c|}
\hline & $\begin{array}{c}\text { Tencur en } \\
\text { matière } \\
\text { sèche \% } \\
\\
\text { Dry } \\
\text { matter } \\
\text { content } \\
\%\end{array}$ & $\begin{array}{c}\text { Teneur } \\
\text { en } \\
\text { cendres } \\
\% \\
\text { Ashes } \\
\%\end{array}$ & $\left|\begin{array}{c}\text { Teneur en } \\
\text { matières } \\
\text { azotées } \\
\text { totales \% } \\
\text { Crude } \\
\text { protein \% }\end{array}\right|$ & $\begin{array}{c}\text { Teneur en } \\
\text { cellulose } \\
\text { brute } \\
\text { Crude } \\
\text { fibre \% }\end{array}$ & $\begin{array}{c}\text { Teneur en } \\
\text { hémicel- } \\
\text { luloses } \\
\text { «Van } \\
\text { Soest } \\
N D F- \\
A D F \\
\text { Van Soest }\end{array}$ & $\begin{array}{c}\text { Teneur en } \\
\text { cellulose } \\
\text { "Van } \\
\text { Soest } \\
\\
A D F- \\
\text { lignin } \\
\text { Van Soest }\end{array}$ & $\begin{array}{c}\text { Teneur en } \\
\text { lignine } \\
\text { \& Van } \\
\text { Soest } ~ \\
\text { Lignin } \\
\text { Van Soest }\end{array}$ \\
\hline $\begin{array}{l}\text { Paille non traitée } \\
\text { Untreated straw }\end{array}$ & 89.1 & 10.3 & 3.5 & 40.8 & 30.9 & 40.6 & 9.2 \\
\hline $\begin{array}{l}\text { Paille traitée } \\
\text { Treated straw }\end{array}$ & 27.5 & 12.9 & 3.1 & 40.8 & 23.2 & 41.9 & 8.9 \\
\hline Maïs - Maize .. & 90.9 & 1.5 & 11.4 & 2.4 & 4.5 & 4.5 & 1.6 \\
\hline Soja-Soyabean & 92.5 & 7.4 & 54.5 & 4.2 & 3.7 & 4.7 & 0.8 \\
\hline
\end{tabular}

\section{Résultats}

\section{A. Quantités ingérées (tabl. 2)}

Les quantités de matière sèche de paille ingérées ont été faibles : en moyenne $25,8 \mathrm{~g}$ de MS/ $\mathrm{kg}$ po.75 pour la paille non traitée et $35,7 \mathrm{~g}$ pour la paille traitée. Le nombre de repas de concentré a eu un effet positif significatif $(+4,7$ p. 100) alors que l'addition de bicarbonate n'en a eu aucun sur les quantités totales ingérées.

\section{B. Digestibilité (tabl. 2)}

La digestibilité de la matière organique des rations a été en moyenne de 58,8 avec la paille non traitée contre 67,7 pour la paille traitée distribuée à volonté. Les valeurs correspondantes pour les hémicelluloses de VAN SoEST (NDF-ADF) ont été de 48,3 et de 66,1 et pour la cellulose de VAN SOEST (ADF-lignine (*)) de 44,0 et de 65,1 . Notons que les digestibilités de la cellulose Weende sont proches de ces dernières $(42,6$ et 60,4$)$. Enfin la digestibilité des matières azotées a été plus faible en présence de paille traitée $(61,0)$ que de paille non traitée $(65,8)$, peut-être à cause d'une reprise plus importante des fermentations avec la paille traitée dans le gros intestin.

(*) Résidus d'une attaque de l'ADF par de l'acide sulfurique pendant 3 heures. 
La distribution du concentré en 4 repas, au lieu de 2 par jour, n'a pas eu globalement d'influence significative sur la digestibilité de la matière organique des rations, bien que cette dernière ait augmenté de 1,5 point en présence de la paille non traitée (soit $+2,5$ points pour la paille seule, si on lui attribue toute l'augmentation). Il y a eu aussi une augmentation pour la ration de paille traitée distribuée en quantité limitée avec du bicarbonate mais il n'y en a eu aucune pour les autres à base de paille traitée. Les tendances observées au niveau de la matière organique sont beaucoup plus nettes au niveau de la digestibilité de la cellulose : pour les rations de paille non traitée respectivement $+4,5$ points pour la cellulose brute, $+4,8$ points pour la cellulose de VAN Soest, mais $-1,8$ point pour les «hémicelluloses 》 de VAN SoEsT. Pour les rations de paille traitée en quantité limitée le passage à 4 repas de concentré a fait baisser la digestibilité de la cellulose en l'absence de bicarbonate et augmenter en sa présence.

L'apport de bicarbonate n'a pas eu non plus d'influence significative sur la digestibilité de la matière organique des rations, ni d'ailleurs sur celle de la cellulose, bien qu'une petite augmentation $(+4,6$ points) soit notée pour la cellulose de VAN SoesT dans les rations de paille non traitée. Par contre l'apport de bicarbonate a fait baisser significativement la digestibilité des hémicelluloses VAN SoEst.

La limitation des quantités distribuées pour les rations de paille traitée a entraîné une augmentation très nette et significative de la digestibilité de leur matière organique, de même pour leurs matières azotées ou leurs parois végétales.

\section{Matière organique digestible (tabl. 2)}

Le nombre de distributions de concentré et l'addition de bicarbonate n'ont pas eu d'influence significative sur les quantités de matière organique digestible ingérées (MOD en $\mathrm{g} / \mathrm{kg} \mathrm{P}^{0.75}$ ). On peut seulement noter une tendance à faire baisser celles-ci lorsque le bicarbonate était associé avec le concentré distribué deux fois par jour.

Les quantités de MOD ingérées par animal et par jour ont donc été en moyenne de $7,3,14,5$ et 12,3 sous forme de paille non traitée, traitée distribuée à volonté et traitée distribuée en quantité limitée.

Enfin la réduction de 30 p. 100 de l'ingestion de la paille traitée n'a entraîné qu'une baisse de 15 p. 100 de sa quantité de MOD ingérée.

\section{- $p H$ (fig. 2)}

D. Caractéristiques du jus de rumen (tabl. 3)

Le $\mathrm{pH}$ a varié largement selon les régimes, de 6,0 à 7,25 lors de la période des prélèvements. Il a été en moyenne de 6,61 pour les régimes de paille non traitée et de 6,52 pour ceux de paille traitée. Avec la paille non traitée on a observé une baisse régulière de ce $\mathrm{pH}$ après le repas du matin pour les 4 régimes alors que, pour la paille traitée, il y a toujours eu un pic aux environs de $9 h$, puis une chute nette.

La distribution du concentré en 4 repas, plutôt que 2, a fait augmenter légèrement. mais significativement, le $\mathrm{pH}$ du jus de rumen des moutons recevant la paille normale $(+0,3$ point), mais pas celui des moutons recevant la paille traitée, sauf lorsqu'elle était distribuée en quantité limitée avec du bicarbonate. 
Tableau 2

Quantités ingérées et digestibilité des pailles étudiées.

\begin{tabular}{|c|c|c|c|c|c|}
\hline & \multirow{2}{*}{$\begin{array}{l}\text { Tampon } \\
\text { Buffer }\end{array}$} & \multirow{2}{*}{$\begin{array}{c}\text { Nombre de } \\
\text { distributions } \\
\text { par jour } \\
\text { Number of } \\
\text { distributions } \\
\text { per day }\end{array}$} & \multicolumn{2}{|c|}{ 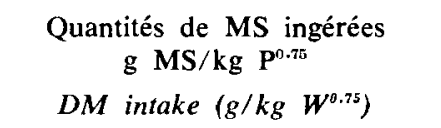 } & \multirow[b]{2}{*}{$\begin{array}{c}\text { Matière } \\
\text { organique } \\
\text { Organic } \\
\text { matter }\end{array}$} \\
\hline & & & $\begin{array}{l}\text { Ration } \\
\text { Diet }\end{array}$ & $\begin{array}{l}\text { Paille } \\
\text { Straw }\end{array}$ & \\
\hline $\begin{array}{l}\text { Paille non traitée } \\
\text { à volonté }\end{array}$ & - & $\begin{array}{l}2 \\
4\end{array}$ & $\begin{array}{l}49.1 \pm 10.3(a b) \\
49.5 \pm 8.8(a b)\end{array}$ & $\begin{array}{l}26.4 \pm 5.7 \\
26.6 \pm 4.6 \\
\end{array}$ & $\begin{array}{l}58.2 \\
59.7\end{array}$ \\
\hline $\begin{array}{l}\text { Untreated straw } \\
\text { ad libitum }\end{array}$ & + & $\begin{array}{l}2 \\
4\end{array}$ & $\begin{array}{l}43.6 \pm 7.1(a) \\
52.5 \pm 8.0(b)\end{array}$ & $\begin{array}{l}23.2 \pm 3.8 \\
27.7 \pm 4.4\end{array}$ & $\begin{array}{l}58.0 \\
59.4\end{array}$ \\
\hline $\begin{array}{l}\text { Paille traitée } \\
\text { à volonté }\end{array}$ & - & $\begin{array}{l}2 \\
4\end{array}$ & $\begin{array}{r}67.7 \pm 15.0(\mathrm{c}) \\
67.5 \pm 3.1(\mathrm{c}) \\
\end{array}$ & $\begin{array}{l}35.4 \pm 8.5 \\
35.8 \pm 1.9 \\
\end{array}$ & $\begin{array}{l}67.7 \\
67.4 \\
\end{array}$ \\
\hline $\begin{array}{l}\text { Treated straw } \\
\text { ad libitum }\end{array}$ & + & $\begin{array}{l}2 \\
4 \\
\end{array}$ & $\begin{array}{l}65.4 \pm 12.8(e) \\
71.0 \pm 16.1(c)\end{array}$ & $\begin{array}{l}34.4 \pm 7.0 \\
37.4 \pm 9.0 \\
\end{array}$ & $\begin{array}{l}68.0 \\
67.5 \\
\end{array}$ \\
\hline $\begin{array}{l}\text { Paille traitée } \\
\text { en quantité limitée }\end{array}$ & - & $\begin{array}{l}2 \\
4 \\
\end{array}$ & $\begin{array}{l}47.1 \pm 0.8 \\
49.2 \pm 0.9 \\
\end{array}$ & $\begin{array}{l}25.0 \pm 0.5 \\
26.1 \pm 0.4 \\
\end{array}$ & $\begin{array}{l}72.9 \\
72.5 \\
\end{array}$ \\
\hline $\begin{array}{l}\text { Treated straw } \\
\text { restricted }\end{array}$ & + & $\begin{array}{l}2 \\
4\end{array}$ & $\begin{array}{l}49.8 \pm 1.1 \\
48.2 \pm 1.0\end{array}$ & $\begin{array}{l}25.9 \pm 0.5 \\
25.1 \pm 0.4\end{array}$ & $\begin{array}{l}70.0 \\
73.0\end{array}$ \\
\hline
\end{tabular}

L'addition de bicarbonate a permis une augmentation systématique significative du $\mathrm{pH}$ dans le cas de la paille normale $(+0,2$ point $)$ mais pas dans celui de la paille traitée, vraisemblablement parce que celle-ci contenait de la soude. La limitation de la quantité de paille traitée offerte n'a pratiquement pas modifié, en moyenne, le pH du jus de rumen.

\section{- Acides gras volatils}

Les teneurs moyennes en acides gras volatils totaux (AGV) ont été de 73,7 mmoles/I pour les 4 régimes de paille non traitée et de 94,3 pour ceux de paille traitée distribuée à volonté.

Le fractionnement de l'apport de concentré n'a pas eu d'effet significatif sur cette teneur en AGV totaux. Par contre, l'apport de bicarbonate l'a légèrement et 
Intake and digestibility of the studied straws.

Digestibility of the diet

Digestibilité de la ration

\begin{tabular}{|c|c|c|c|c|c|c|}
\hline & \multirow{3}{*}{$\begin{array}{c}\text { calculee } \\
\text { de la paille } \\
\\
\text { Calculated } \\
\text { digestibility } \\
\text { of the straw } \\
\text { (organic matter) }\end{array}$} & \multirow{2}{*}{\multicolumn{2}{|c|}{$\begin{array}{c}\mathrm{g} / \mathrm{kg} \mathrm{P}^{0.75} \\
\text { Intake of digestible } \\
\text { organic matter } \\
\mathrm{g} / \mathrm{kg} W^{0.75}\end{array}$}} \\
\hline \multirow{2}{*}{$\begin{array}{c}\text { Matières } \\
\text { azotées } \\
\text { totales } \\
\text { Crude } \\
\text { protein }\end{array}$} & \multirow{2}{*}{$\begin{array}{l}\text { Cellulose } \\
\text { brute } \\
\text { Crude } \\
\text { fibre }\end{array}$} & \multirow{2}{*}{$\begin{array}{l}\text { Hémicellu- } \\
\text { loses } \\
\text { Van Soest } \\
\text { Hemicellu- } \\
\text { loses } \\
\text { Van Soest }\end{array}$} & \multirow{2}{*}{$\begin{array}{l}\text { Cellulose } \\
\text { Van Soest } \\
\text { Cellulose } \\
\text { Van Soest }\end{array}$} & & & \\
\hline & & & & & $\begin{array}{c}\text { Ration } \\
\text { Diet }\end{array}$ & $\begin{array}{l}\text { Paille } \\
\text { Straw }\end{array}$ \\
\hline 65.0 & 39.5 & 52.5 & 38.9 & 30.0 & 26.1 & 7.1 \\
\hline 66.8 & 44.8 & 49.8 & 44.4 & 32.3 & 27.0 & 7.9 \\
\hline 66.1 & 41.3 & 46.0 & 44.2 & 30.3 & 24.1 & 6.3 \\
\hline 65.4 & 45.0 & 45.0 & 48.4 & 32.3 & 28.1 & 8.1 \\
\hline 60.8 & 60.5 & 68.5 & 64.9 & 46.0 & 41.2 & 14.2 \\
\hline 60.3 & 59.4 & 68.8 & 64.0 & 46.1 & 41.1 & 14.4 \\
\hline 62.3 & 61.3 & 65.2 & 65.8 & 47.5 & 39.6 & 14.2 \\
\hline 60.4 & 60.3 & 62.1 & 65.7 & 46.2 & 42.9 & 15.1 \\
\hline 66.8 & 73.7 & 82.1 & 74.5 & 57.4 & 30.8 & 12.6 \\
\hline 66.1 & 68.2 & 80.6 & 70.5 & 56.0 & 32.1 & 12.7 \\
\hline 65.5 & 68.2 & 69.8 & 68.7 & 51.2 & 30.9 & 11.4 \\
\hline 68.6 & 71.5 & 81.8 & 73.5 & 57.0 & 31.1 & 12.6 \\
\hline
\end{tabular}

significativement augmenté ( +7 p. 100) surtout quand le concentré était distribué en deux repas.

La limitation de la quantité de paille traitée offerte a fait baisser la teneur en AGV totaux du jus de rumen de 94,3 à 82,9 mmoles $/ 1$.

En fait, la teneur en AGV totaux a essentiellement varié dans le même sens que la quantité de MOD ingérée :

$$
\mathrm{AGV}_{(\text {mmoles } / 1)}=42,9+1,24 \text { MODI }\left(\mathrm{g} / \mathrm{kg} \mathrm{P}^{0.75}\right) \quad \mathrm{n}=12 \quad \mathrm{r}=0,762
$$

Le pourcentage d'acide acétique a été plus faible pour la paille non traitée : 65,2 p. 100 contre 69,1 et 70,6 p. 100 pour les pailles traitées, à volonté et en quantité limitée. Le fractionnement des apports de concentré et l'addition de bicarbonate n'ont eu, globalement, aucun effet significatif sur les proportions des différents acides gras volatils. 


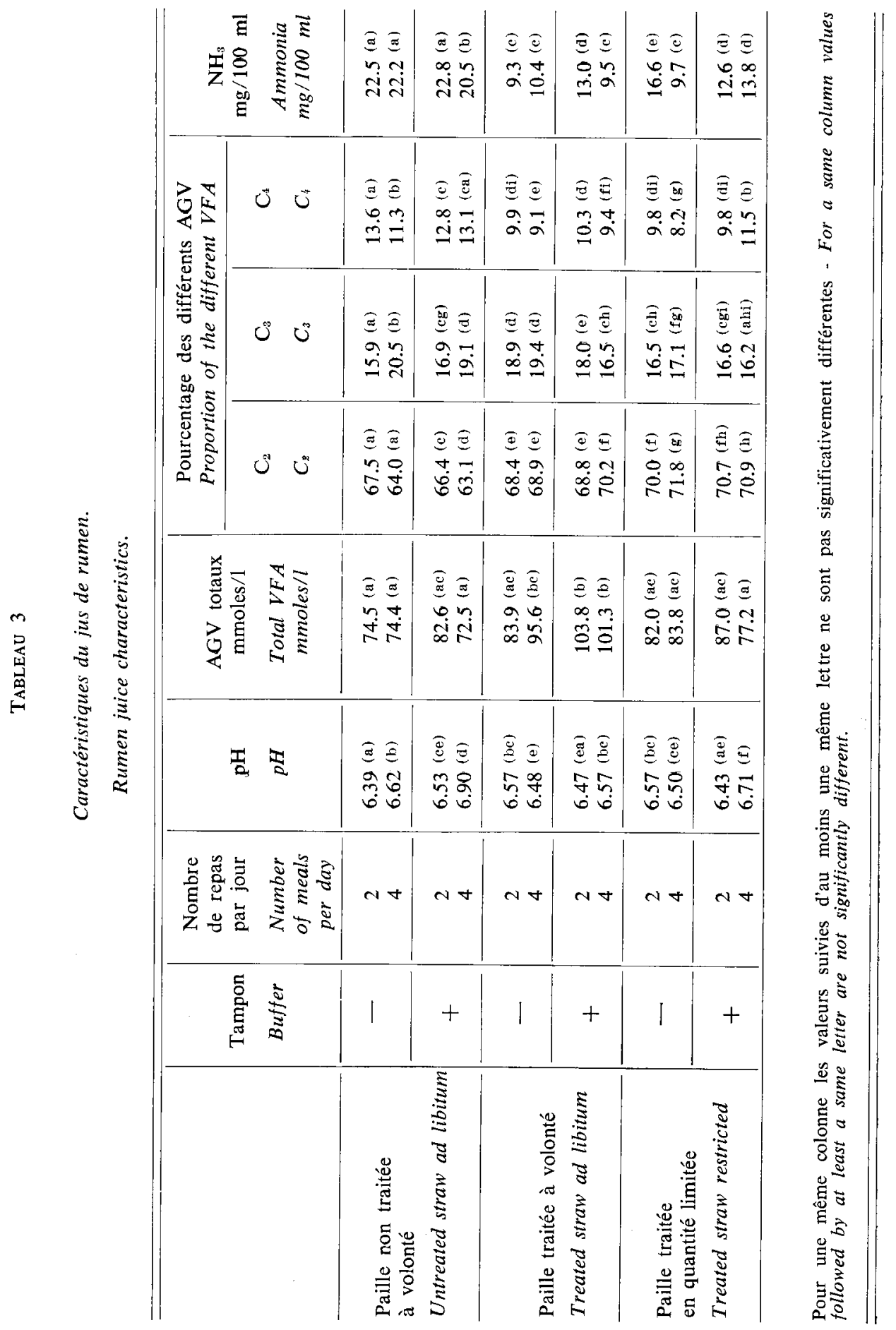




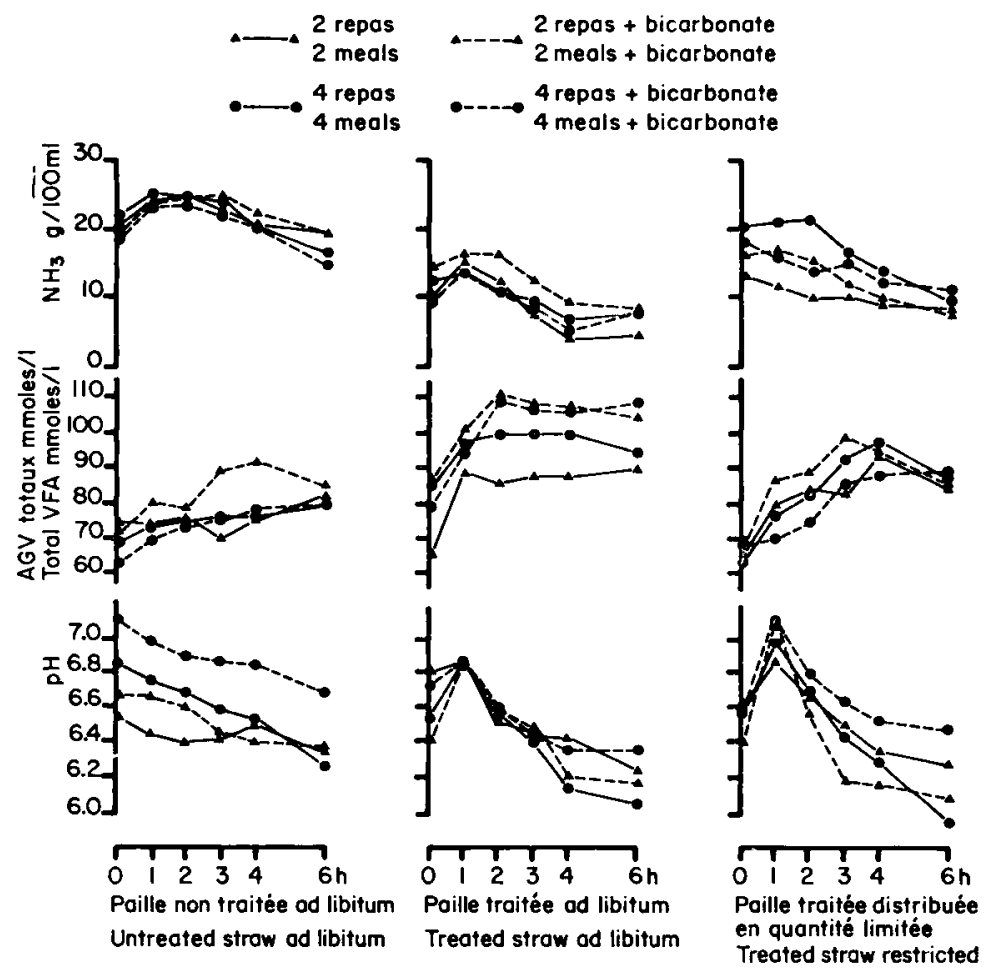

FIG. 2

Caractéristiques du jus de rumen.

Rumen juice characteristics.

- $\mathrm{NH}_{3}$ (tabl. 3)

La teneur en $\mathrm{NH}_{3}$ du jus de rumen a varié, selon les régimes, de 5 à $25 \mathrm{mg} / 100 \mathrm{ml}$. Elle a été en moyenne de 22 pour les régimes à base de paille non traitée, 10,6 et 13,2 pour ceux à base de paille traitée offerte à volonté et en quantité limitée.

Le fractionnement des repas a eu tendance à faire baisser la teneur en $\mathbf{N H}_{3}$ (-11 p. 100), mais non significativement. L'addition de bicarbonate n'a eu par contre aucun effet.

En définitive, la teneur en $\mathrm{NH}_{3}$ du jus de rumen a varié essentiellement en sens inverse de la MOD ingérée :

$$
\mathrm{NH}_{3}(\mathrm{mg} / 100 \mathrm{ml})=38,4-0,704 \text { MODI } \mathrm{n}=12 \quad \mathrm{r}=-0,852
$$

\section{Discussion}

Notre objectif était de limiter partiellement la mauvaise utilisation des pailles en présence d'une quantité d'amidon élevée. 
La limitation du niveau alimentaire a augmenté de façon importante la digestion des parois de la paille traitée à la soude et donc la digestibilité de la matière organique de cette paille. Nous avons déjà étudié et discuté cet effet par ailleurs (Dulphy ot al, 1982 a et 1983). MaENG, Mowat \& Bilanski (1971) l'ont aussi montré en utilisant un protocole d'étude tout à fait différent du nôtre.

Les deux autres techniques étudiées, fractionnement de lapport de concentré et additicn de bicarbonate de soude, ne se sont, par contre, guère révélées efficaces.

Le fractionnement de l'apport de concentré a augmenté légèrement les quantités totales ingérées, peut-être grâce à une légère amélioration de la vitesse de digestion dans le rumen. Globalement, l'effet de ce fractionnement n'est pas significatif sur les autres paramètres enregistrés. Avec la paille non traitée on note cependant une tendance à l'augmentation de la digestibilité de la cellulose brute et donc de la matière organique de la paille. Cette tendance est d'ailleurs parallèle à une élévation du $\mathrm{pH}$. Cependant la légère amélioration de la digestion de cette paille peut être due à une diminution de la quantité d'amidon en cours de digestion dans le rumen à un instant donné. En effet, lorsque les animaux ingèrent une quantité importante d'amidon ce sont plus ses produits de dégradation que le faible $\mathrm{pH}$ qui inhibent l'activité des cellulases et des hémicellulases du rumen (HenNing et al., 1980). Il est donc probable que l'effet dépressif de l'amidon sur la digestion des pailles puisse être atténué en étalant plus son apport sur 24 heures ou en le mélangeant à la paille.

Le bicarbonate de sodium est recommandé depuis longtemps pour maintenir ou améliorer l'ingestion des rations riches en céréales (Kellaway et al., 1977 ; Erdman et al., 1980 ; Wheeler \& Noller, 1976 ; WheEler et al., 1980), limiter la chute du taux butyreux (EMERY \& B: own, 1961 ; EMERY, Brown \& ThOMAS, 1964) ou s'opposer à certains troubles tels que l'acidose (DunN, EMERICK \& EMBry, 1979). Cependant dans notre essai l'apport de céréales a été faible non seulement en proportion (30 p. 100 seulement de maïs-grain), mais en quantité à cause de la faible ingestibilité de la paille. Cela doit expliquer l'absence d'effet du bicarbonate sur les quantités ingérées. Il y a eu absence d'effet aussi au niveau des autres paramètres étudiés sauf une baisse inexplicable de la digestibilité des hémicelluloses de VaN Soest et une augmentation, aussi peu explicable, de la teneur en AGV du jus de rumen. Enfin, Ie bicarbonate a bien fait augmenter le $\mathrm{pH}$ du jus de rumen, mais seulement avec la paille non traitée et il n'y a eu aucune répercussion sur la digestibilité de cette paille. Le $\mathrm{pH}$ était cependant demeuré dans des limites normales, même sans bicarbonate et son action sur la digestion des parois végétales n'est pas toujours déterminante, comme nous l'avons noté plus haut.

Avec le bicarbonate, OsBourn et al. (1970) ont noté un effet positif net sur le pH du jus de rumen et son activité cellulolytique, mais le bicarbonate représentait environ 10 p. 100 de la ration. Nous n'avons utilisé qu'une dose de 1,7 p. 100 pour la paille normale et de 1,2 p. 100 pour la paille traitée à volonté. Cette dose a aussi été utilisée par ERDMAN et al. (1980) avec succès, mais pour une ration plus riche en céréales. Dans le cas qui nous intéresse, il reste donc à savoir si le bicarbonate a vraiment un effet négligeable ou si, en augmentant sa dose, il pourrait permettre le maintien d'une digestion correcte des parois cellulosiques des pailles. Cette dernière hypothèse n'est pas du tout certaine car le $\mathrm{pH}$ du jus de rumen, dans nos essais, n'a jamais été très bas. Il est même relativement élevé avec la paille traitée à la soude. L'intérêt du bicarbonate semble donc pratiquement nul pour le type de ration que nous avons étudiée. 
La comb:naison des trois techniques que nous avons utilisées n'a eu aucun effet clair, par contre l'effet de l'étalement de la distribution du concentré a été plus net en présence de bicarbonate qu'en son absence.

Enfin, les teneurs en acides gras volatils et en ammoniac du jus de rumen ne semblent pas avoir été modifiées directement par les différents régimes étudiés, mais bien indirectement. En effet, lorsque la quantité de matière organique digestible ingérée a augmenté, la teneur en acides gras volatils du jus de rumen a également augmenté et celle en ammoniac a diminué, traduisant tout simplement une activité plus importante de la microflore du rumen.

Accepté pour publication en novembre 1982.

\section{Summary \\ Feeding value of sodium hydroxide treated or untreated cereal straw. $I V$. Influence of the number of concentrate meals and the addition of sodium bicarbonate}

The feeding value of untreated or sodium-hydroxide treated straw decreases when the proportion of concentrates in the diet, particularly cereals, increases. To limit this reduction, the following three techniques were used separately, then together :

- restriction of straw supply;

- increase in the number of concentrate meals ;

- addition of sodium bicarbonate $(20 \mathrm{~g} /$ animal/day $)$.

To this purpose, a straw before and after treatment was given to sheep together with 45 p. 100 concentrates (fig. 1, tabl. 1).

The increased concentrate feeding only allowed a slight but significant $(+4.7$ p. 100) improvement in voluntary straw intake (tabl. 2).

Fecd restriction led to a very marked improvement in treated straw digestibility (tabl. 2).

Increased concentrate feeding slightly modified the digestibility of the diets $(+1.5$ point $)$ but not significantly (tabl. 2).

Addition of sodium bicarbonate had no significant effect on diet digestibilities (tabl. 2).

Rumen juice characteristics $\left(\mathrm{pH}, \mathrm{VFA}, \mathrm{NH}_{\mathrm{i}}\right.$ ) for the different diets were measured (tabl. 3. fig. 2).

The poor digestion of straw given together with a large proportion of starch was only partly prevented by reducing the straw supply. Results are discussed.

\section{Références bibliographiques}

Dulphy J.P., Breton J., Bienaimé A., Louyot J.M., 1982 a. Etude de la valeur alimentairc des pailles de céréales traitées ou non à la soude. I. - Influence du traitement à la soudle. Ann. Zootech., 31, 195-214.

Dulphy J.P., Kouassi B., Bienaimé A., 1982 b. Etude de la valeur alimentaire des pailles de céréales traitées ou non à la soude. II. - Influence de la nature du complément énergétique. Ann. Zootech., 31, 215-232.

Dilphy J.P., Breton J., Louyot J.M., Bienaimé A., 1983. Etude de la valeur alimentaire des pailles de céréales traitées ou non à la soude. III. - Influence du niveau d'apport d'aliment concentré. Ann. Zootech., 32 (1), 55-81. 
DunN B.H., Emerick R.J., Embry L.B., 1979. Sodium bentonite and sodium bicarbonate in high concentrate diets for lambs and steers. J. Anim. Sci., 48, 764-769.

EMERY R.S., BROWN L.D., 1961. Effect of feeding sodium and potassium bicarbonate on milk fat, rumen $\mathrm{pH}$ and volatile fatty acid production. J. Dairy Sci., 44, 1899-1902.

EMERY R.S., BROWN L.D., ThOMas J.W., 1964. Effect of sodium and calcium carbonates on milk production and composition of milk, blood and rumen contents of cows fed grain ad libitum with restricted roughage. J. Dairy Sci., 47, 1325-1329.

Erdman R.A., Botrs R.L., Hemken R.W., Bull L.S., 1980. Effect of dietary sodium bicarbonate and magnesium oxide on production and physiology in early lactation. J. Dairy Sci., 63, 923-930.

Henning P.A., Van der Linden Y., Mattheyse M.E., Nauhaus W.K., Schwartz H.M., 1980. Factors affecting the intake and digestion of roughage by sheep fed maize straw supplemented with maize grain. J. Agric. Sci. Camb., 94, 565-573.

Kellaway R.C., Thomson D.J., Beever D.E., Osbourn D.F., 1977. Effects of $\mathrm{NaCl}$ and $\mathrm{NaHCO}$, on food intake, growth rate and acide-base balance in calves. J. Agric. Sci. Camb., 88, 1-9.

Maeng W.J., Mowat D.N., BILANSKi W.K., 1971. Digestibility of sodium hydroxydetreated straw fed alone or in combination with alfalfa silage. Can. J. Anim. Sci., 51, 743-747.

Osbourn D.F., Terry R.A., Cammell S.B, Outen G.E., 1970. Some effects of feeding supplements of maize meal and sodium bicarbonate upon digestion of forage cellulose by sheep. Proc. INutr. Soc., 29, 12 A (Abstr.).

Wheeler W.E., Noller C.H., 1976. Limestone buffers in complete mixed rations for dairy cattle. J. Dairy Sci., 59, 1788-1793.

Wheeler T.B., Wangsness P.J., Muller L.D., Griel Jr. L.C., 1980. Addition of sodium bicarbonate to complete pelleted diets fed to dairy calves. J. Dairy Sci., 63, 18551863. 